\title{
Entrelacs
}

Cinéma et audiovisuel

Hors-série $n^{\circ} 4$ | 2016

Paysages en séries

\section{True Detective et la nature de Louisiane comme impasse}

True Detective et la nature de Louisiane comme impasse

\section{Maylis Asté}

\section{(2) OpenEdition}

Journals

\section{Édition électronique}

URL : http://journals.openedition.org/entrelacs/2135

DOI : 10.4000/entrelacs.2135

ISBN : $2261-5482$

ISSN : 2261-5482

Éditeur

Éditions Téraèdre

Édition imprimée

Date de publication : 1 novembre 2016

ISSN : 1266-7188

\section{Référence électronique}

Maylis Asté, «True Detective et la nature de Louisiane comme impasse », Entrelacs [En ligne], Hors-

série $n^{\circ} 4$ | 2016, mis en ligne le 06 février 2018, consulté le 04 mai 2019. URL : http://

journals.openedition.org/entrelacs/2135; DOI : 10.4000/entrelacs.2135

Ce document a été généré automatiquement le 4 mai 2019.

Tous droits réservés 


\section{True Detective et la nature de Louisiane comme impasse}

True Detective et la nature de Louisiane comme impasse

Maylis Asté

1 La mémoire de la Louisiane se façonne tout comme le maillage de ses côtes: sans frontière nette entre la terre et l'eau. Nic Pizzolatto, créateur de la série True Detective (2014) a grandi dans le sud-ouest de cet Etat. Il déclare avoir été viscéralement touché par ce territoire ${ }^{1}$ dont il présente la nature riche et complexe.

2 Le vaudou et les démons de Angel Heart ${ }^{2}$, les paysages grandioses de Louisiana story ${ }^{3}$ ou la fièvre tropicale d'Un tramway nommé désir ${ }^{4}$ participent de la mythologie des lieux. A l'heure actuelle, la multiplication des productions audiovisuelles en Louisiane est facilitée par une politique de réduction d'impôt ainsi que la construction d'un imposant studio à la Nouvelle Orléans. Toutefois, ce qui attire avant tout les tournages, c'est la présence de décors naturels uniques. Ces dernières années, la série prenant le relais du cinéma, cinq productions à large diffusion ont pour cadre le territoire louisianais. En effet, les séries True Blood (2008), Treme (2010), American Horror Story: Coven (2013), The Originals (2013) et True Detective (2014) ont toutes pour cadre l'État de Louisiane.

3 La nature «sauvage » louisianaise fait rêver : elle propose une atmosphère propice aux élans fantasmagoriques. Le cinéma de genre, et particulièrement les représentations du vampirisme, s'épanouissent dans l'imaginaire attaché à la Louisiane. Dans ses travaux, Morgane Caussarieu ${ }^{5}$ interprète le voyage du mythe vampirique, des conifères des Carpates aux cyprès des bayous, comme une expression du malaise et de la culpabilité du vieux sud, esclavagiste et puritain.

Le cyprès chauve des bayous bénéficie d'un système racinaire double qui lui permet à la fois de respirer et de garantir sa stabilité. Tout comme cet arbre emblème, exposant à la vue de tous ses racines aériennes, les louisianais, ancrés sur un territoire à risque, sont fiers de montrer leurs traditions, leur héritage culturel.

5 L'évocation de la Nature s'avère fondatrice dans la construction de la représentation sociale $^{6}$ attachée au territoire louisianais. La série True Detective - comme, avant elle et 
dans un tout autre registre, la série True Blood - se déroule dans le milieu rural, un espace souvent marginalisé. La vision des campagnes louisianaises qu'elle propose est celle d'une terre d'appartenances plurielles; les hommes possèdent la terre tout comme elle les possède. Nic Pizzolatto crée des personnages qui entretiennent un rapport intime mais ambivalent avec leur territoire: ils y sont engoncés, enfermés. En quoi la nature louisianaise permet-elle la représentation de cette impasse?

\section{La Nature Physique : Impasse géographique et sociale}

6 L'auteur de la série True Detective a soin de replacer ses personnages dans un environnement social, géographique et historique précis qui contribue à la création d'une atmosphère singulière. Plongés au cœur d'un espace complexe où rien n'altère la moiteur ambiante ${ }^{7}$, les personnages de True Detective se trouvent acculés. La nature louisianaise fait figure d'étau, une impasse tant géographique qu'humaine qui représente un danger pour tous ceux qui la parcourent. Entre 1723 et 1986, la Louisiane est balayée par pas moins de huit cent quatre-vingt cinq ouragans et tornades ${ }^{8}$, d'après la Louisiana Geological Survey. Ces catastrophes naturelles ont des conséquences sociales et économiques terribles. La figure de l'impasse, définie comme une position ou une situation ne présentant aucune issue favorable, revient à chaque strate temporelle du récit.

De chasseur-cueilleur caractérisé par le nomadisme, l'Homme se sédentarise peu à peu. La révolution néolithique est un pas décisif dans l'histoire de l'humanité. Elle est favorisée par l'acquisition d'un pouvoir de l'homme sur la Nature: il pratique désormais l'agriculture et l'élevage. La maîtrise de l'homme sur son milieu est encore modeste car il demeure soumis au climat et aux catastrophes naturelles. Toutefois, il se sent investi d'une mission de civilisation de la nature sauvage. L'homme tente de rationaliser son environnement, il s'ancre dans un territoire qu'il fait sien. La sédentarité lui permet d'accumuler richesse et savoir, dans un rapport intime avec un espace vécu.

La série True Detective présente toute une galerie de personnages enracinés, pour qui la seule unité géographique semble être "around here »". Ces hommes et femmes sont présentés comme des survivants, ceux qui sont restés après les catastrophes naturelles et ceux qui n'ont pas encore succombé aux diverses maladies favorisées par la pollution généralisée. Ces personnages ne se meuvent que par un vieil instinct de survie, ils n'évoluent plus, ne se renouvellent pas, ils n'ont pas d'héritage à transmettre. La sédentarité parait ainsi porteuse d'une rupture dans la circulation des savoirs.

Le sentiment de l'impossibilité du legs est renforcé par le fait que ces personnages sont souvent apparentés aux victimes : si les enfants disparaissent, à qui transmettra-t-on un héritage ? À l'image de Danny Fontenot ${ }^{10}$, une ancienne star du baseball recroquevillée dans un canapé et incapable d'articuler un mot, les survivants restent figés, sans même avoir la force de se révolter face à la disparition de leurs enfants. 


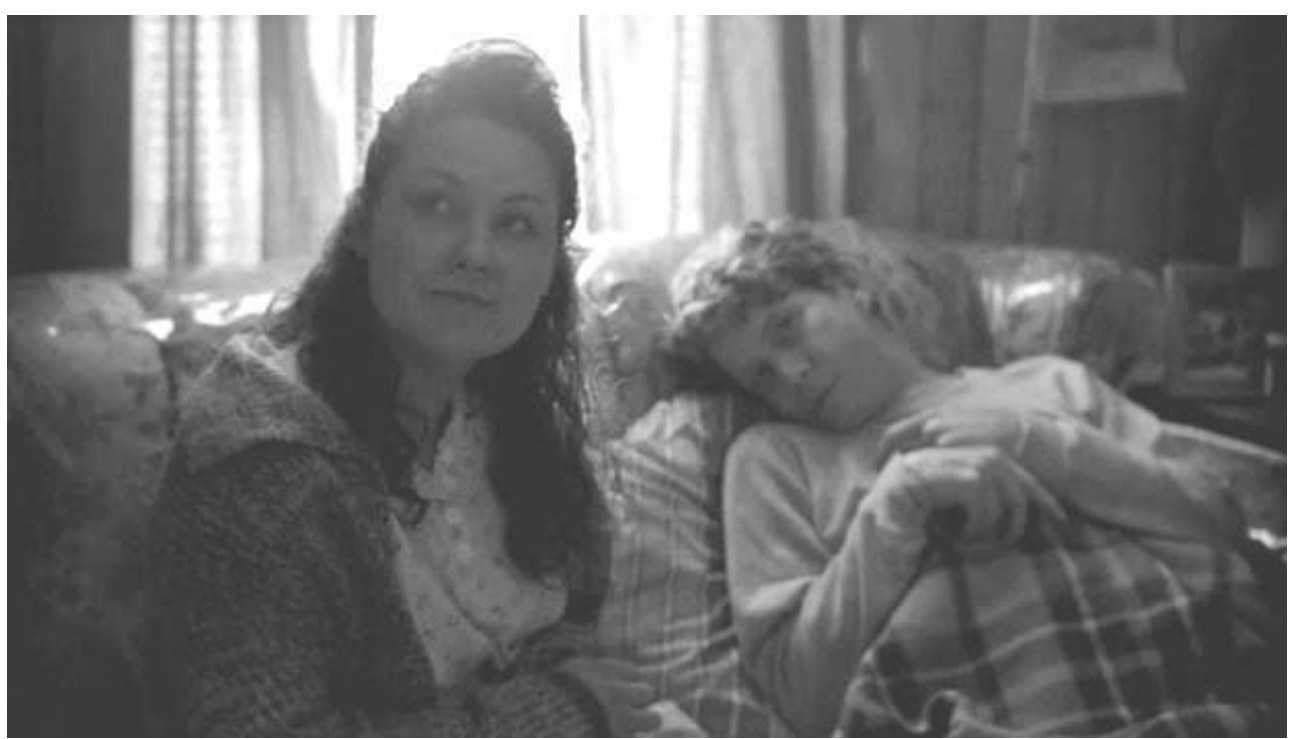

Fig. 1 - Danny Fontenot S1.Ep.01

10 La sédentarité proposée par la majorité des personnages de True Detective est une forme d'atavisme dégénératif. Figure de cette dimension pathogène du lieu, la mère de Dora Lange ${ }^{11}$, Mme Kelly, a les mains qui tremblent et les ongles détruits. Après vingt ans de travail dans une teinturerie en contact avec des produits chimiques, elle souffre également de migraines chroniques. À son image, l'individu enraciné n'est plus force de proposition; il perpétue à la rigueur quelques traditions en attendant la fin. Ces figures sont autant de peintures de la stase. Rustin Cohle affirme d'ailleurs : "People out here, it's like they don't even know the outside world exists ${ }^{12}$.

11 La série présente la désagrégation de la famille nucléaire. La seule famille soudée au début de la série est celle de Martin, cependant elle ne résistera pas à l'usure du temps. Le paysage social décrit par Nic Pizzolatto correspond à celui des « white trash ${ }^{13}$ ». Poussant à l'extrême la représentation d'un espace pétrifié, la série aborde, à différentes reprises, le thème de l'inceste. Dès l'épisode $2^{14}$, deux personnages (dont la mère de Dora Lange) mentionnent des relations incestueuses, auxquelles s'ajoutent les rapports consanguins qu'Errol Childress entretien avec sa sœur ${ }^{15}$. Symbole majeur de décadence, l'inceste est l'un des stigmates d'un espace forclos. La fin de la lignée, de la race, est une nouvelle impasse. Ce terme, traduit en anglais par la formule «dead end " exprime une forte connotation mortifère.

12 La société globalisée actuelle valorise la mobilité, la flexibilité. Ceux qui ne participent pas de ces flux perpétuels - forme de retour au nomadisme - sont perçus comme des populations archaïques. Ils appartiennent à un lieu, à un paysage, sans jamais avoir le recul suffisant pour s'en abstraire. À l'immobilisme des enracinés s'oppose le personnage de Rustin Cohle, figure d'un ailleurs. D'origine texane, il a vécu en Alaska, puis est retourné au Texas, avant d'être affecté en Louisiane. Au cours d'une séquence de bal traditionnel ${ }^{16}$, il suscite l'admiration de sa cavalière en évoquant Paris, ville où il a séjourné durant un mois. La tête dans les étoiles, Rustin incarne le nomadisme contrairement à Martin Hart, qui représente la stabilité, les racines. Par sa mise en scène, Cary Fukunaga joue de cette dichotomie entre mouvement et fixité. Ce motif symbolique inscrit dans le paysage est repris périodiquement. Ainsi à la fin de l'épisode $2^{17}$, un plan d'ensemble présente une église délabrée, envahie par la végétation, alors qu'à l'arrière 
plan de ce lieu en déshérence passe un train de marchandise. L'épisode 7, quant à lui, se conclut par un autre plan large où l'on voit un cimetière perdu dans un trou de verdure, tandis qu'à l'arrière plan un bateau glisse sur le fleuve. Le mouvement est aussi associé aux voitures et aux investigations de Martin Hart et Rustin Cohle.

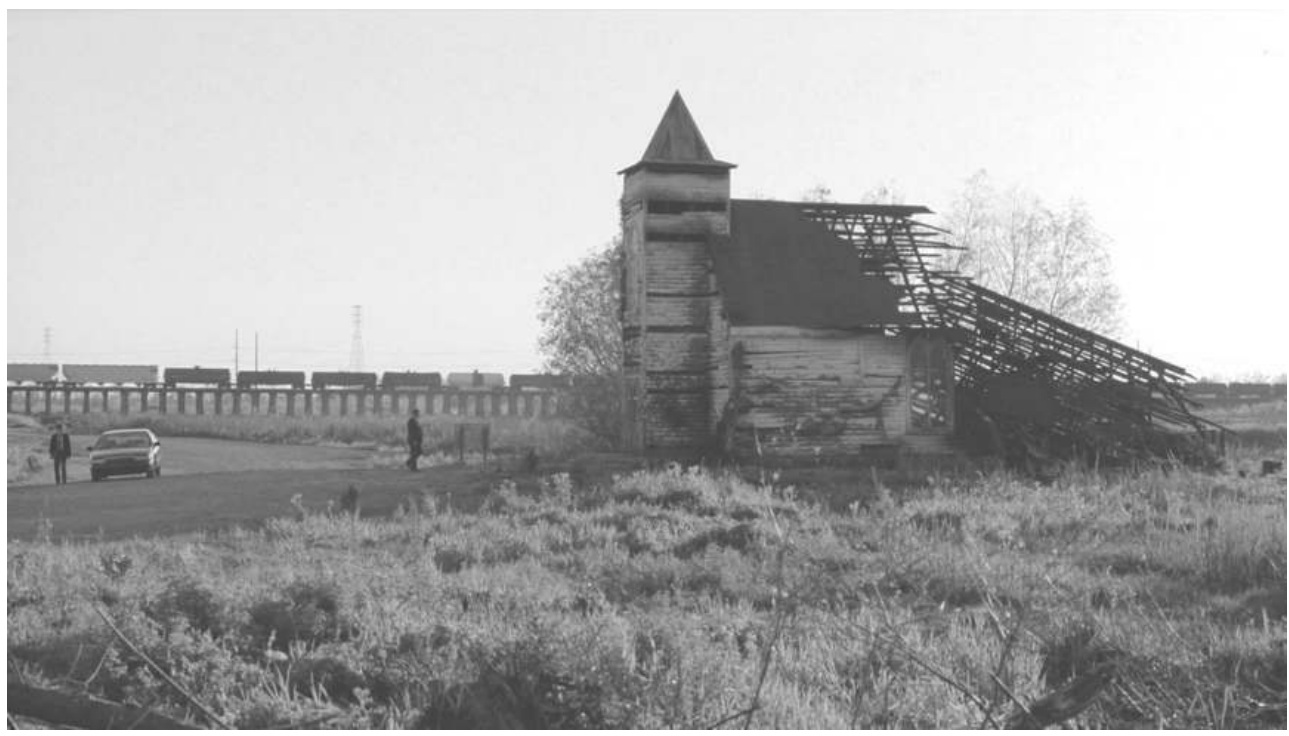

Fig.2 S1.Ep.02

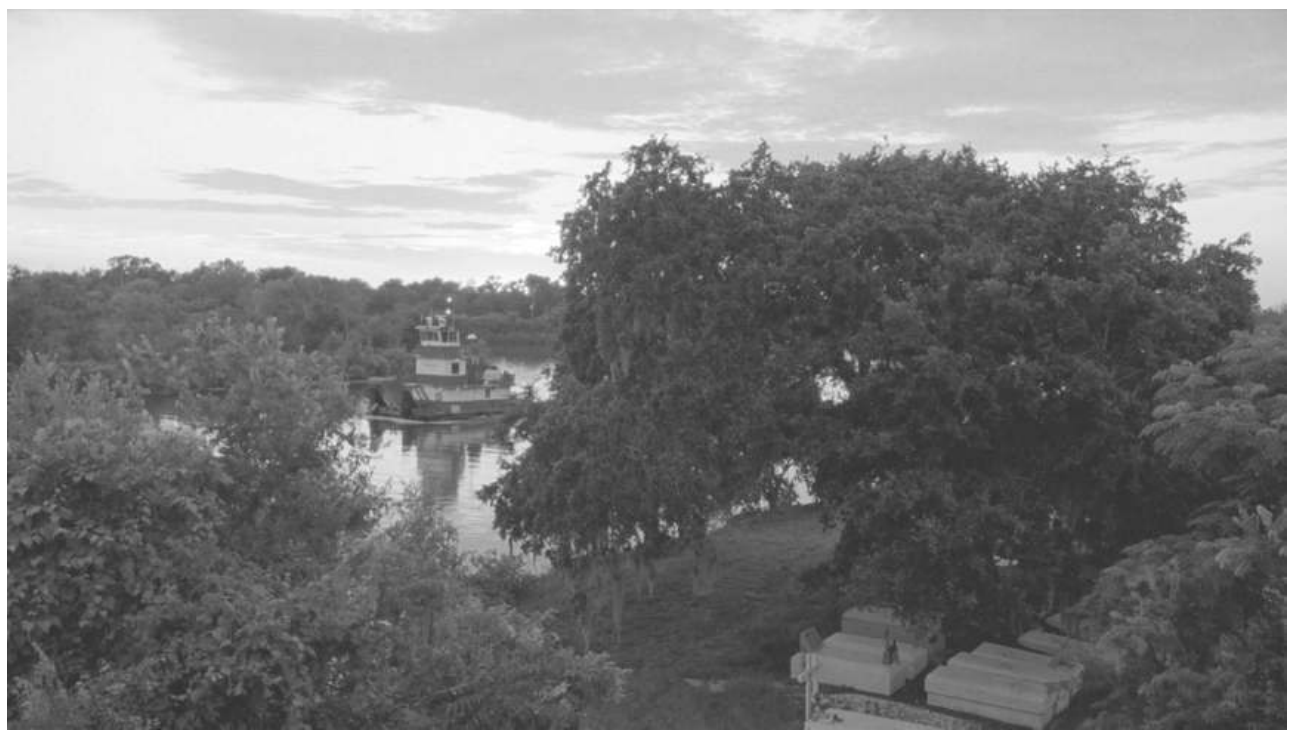

Fig.3 S1.Ep.07

Le milieu dans lequel Martin Hart et son partenaire évoluent est un espace verrouillé. Celui-ci figure l'impasse tout en étant lui même en sursis. En effet, comme le souligne Nic Pizzolatto ${ }^{18}$, le sud de la Louisiane risque d'être englouti d'ici quinze à vingt ans. La Nature qui s'impose aux personnages de la série est hybride - à la fois sauvage et altérée par la main de l'homme. Toutefois, une tendance nette à "l'ensauvagement» est perceptible. Les espaces-refuges envahis par les végétaux habités par Reggie Ledoux et Errol Childress sont représentatifs de cette dynamique. La nature enferme, autant qu'elle offre un lieu d'asile à ces personnages monstrueux. En ce sens, elle assume un rôle ambiguë. 

le monde végétal. La Nature construite et reconstruite par l'homme ${ }^{19}$ répond aux normes que celui-ci se choisit: ces normes sont autant de frontières qui s'emboîtent. Dans l'épisode pilote, Martin Hart, en évoquant les maisons éparses des campagnes louisianaises qu'ils sont en train de traverser, affirme: "There's all kinds of ghettos in the world.", ce à quoi Rustin renchérit: "It's all one ghetto ${ }^{20}$. Cette représentation d'un monde qui n'est qu'enclaves, à diverses échelles, est un motif récurrent dans la série. Lorsque ce n'est pas la nature environnante qui enferme l'homme, c'est l'homme luimême qui érige des murs. par le biais de pénitenciers, d'hôpitaux psychiatriques ou d'écoles. Les établissements scolaires étant dans la série non pas des lieux d'épanouissement personnel, mais le lieu $\mathrm{du}$ traumatisme, du contre-nature ${ }^{21}$. Ces écoles mènent soit à la mort, soit directement aux deux autres institutions. Par ailleurs, il est révélateur que la séquence d'affrontement final entre Errol Childress et Rustin Cohle ait lieu dans un fort à l'abandon. En effet, le fort est, architecturalement parlant, l'emblème de l'enclave. La clôture de cet espace est accentuée par l'invasion d'une végétation luxuriante. L'édifice ainsi que son environnement naturel propose un jeu d'emboîtement. appartient à qui ?), révèle un besoin identitaire autant que l'affirmation de la virilité des personnages masculins. Dans True Detective la Nature en tant qu'espace et la Nature en tant qu'essence ${ }^{22}$ contribuent à part égale à la pétrification des hommes. Ainsi se forme peu à peu l'image du piège. Les petites constructions de bois faites par les enfants, que le révérend ${ }^{23}$ présente comme des « pièges à oiseaux » ou « filets du diable » figurent dans la série cette symbolique du piège.

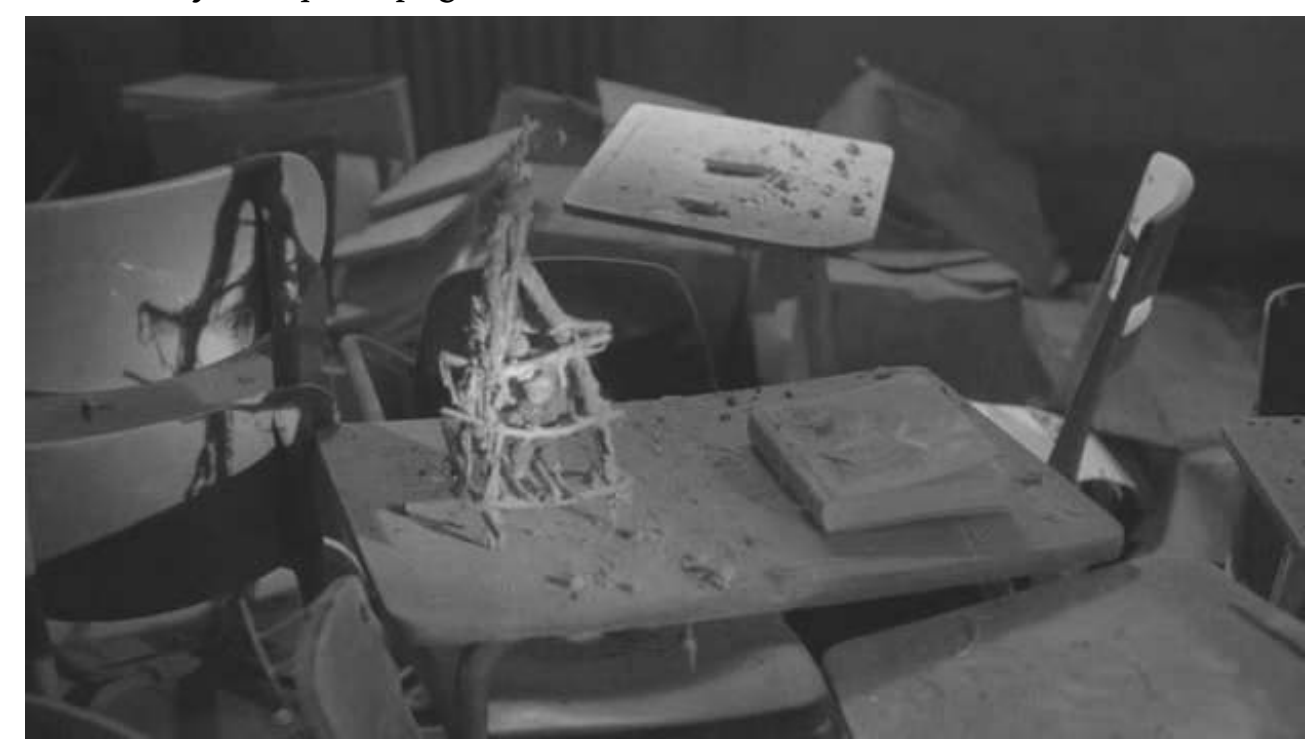

Fig 4 : Bird Trap

Cet enfermement dans un espace, dans une communauté ou en soi-même (par les diverses évocations de la folie) fait de l'homme une espèce en péril. Pourtant, dans True Detective, il est le seul représentant de la riche faune de Louisiane. Il semble, dans la série, que la Nature ait éradiqué tous les autres animaux, ou que l'homme se soit imposé comme le prédateur suprême. Les animaux ne sont d'ailleurs présents que grâce au travail des 
taxidermistes. Ils servent de décor aux bureaux ou aux intérieurs de maison : l'animal devient une pièce de musée ou un trophée.

La Nature en tant qu'écosystème est en péril. Si la chasse est une activité très répandue en Louisiane, les deux parties de chasse représentées dans True Detective sont consacrées à des chasses à l'homme. En cela, Rustin Cohle est un digne héritier de la figure mythique $\mathrm{du}$ «Coureur des bois». En tant que détective il suit des traces, découvre des pistes : il traque, sur des espaces immenses, le monstre aux oreilles vertes.

19 L'Homme, animal survivant, se protège par le repli dans un univers de la clôture. Les bureaux de police, les voitures ou les arrière-cours sont autant d'emblèmes de cet enfermement. Ce sentiment est appuyé par le recours à de nombreux surcadrages qui participent de la fragmentation des espaces.

\section{La Nature Métaphysique : Une impasse pour l'humanité}

20 La nature humaine ${ }^{24}$ entretient des rapports conflictuels avec la civilisation et ses cadres ; elle semble perpétuellement en butte à des problématiques existentielles. Telle que la conçoit Rustin ${ }^{25}$, la nature humaine n'offre aucune possibilité de rédemption. L'homme est pétrifié, or la rédemption sous-entend un mouvement volontaire. Dépourvu de fin, l'être humain est une créature absurde, le jouet d'une entité supérieure.

21 Face à l'impasse, les hommes vont chercher refuge dans l'illusion, au sens de perception erronée et ne correspondant pas à la réalité dite «objective». Dans True Detective, la première de toutes les illusions-refuge est celle que créent les produits modificateurs de conscience. Les substances psychotropes, tant l'alcool que les autres drogues dites «dures » (méthamphétamine, cocaïne, etc.), apparaissent comme une échappatoire, un moyen de fuir sa nature ou sa «programmation ${ }^{26}$ » comme le dit Rustin. Ce mirage de libération est en définitive une nouvelle impasse : celle de l'addiction.

La série présente la drogue comme une réponse aux angoisses métaphysiques. La grande majorité des personnages de la série boivent: beaucoup de policiers sont présentés comme des alcooliques, Martin Hart boit lorsqu'il culpabilise d'avoir trompé sa femme et Rustin Cohle boit le jour anniversaire de la mort de sa fille, puis se présente lui-même comme un alcoolique en 2012. Le pasteur revivaliste ${ }^{27}$ Theriot, quand Rustin l'interroge en 2002, a sombré dans l'alcoolisme. Il explique : « All my life I wanted to be nearer to God, but the only nearness: silence $»^{28}$. L'alcoolisme est pour lui un refuge face à l'absence de sens du monde : c'est à ce vide existentiel qu'il tente d'échapper. La dépendance à l'alcool du pasteur est présentée par le révérend Tuttle comme une perte de maîtrise, une dégénérescence. Il souligne : "It's hard to trust a man who can't trust himself with a beer ${ }^{29}$ ".

La drogue permet de créer un monde artificiel, par définition un monde en dehors de la Nature. Les hallucinations visuelles de Rustin sont représentées à l'écran et nous permettent de percevoir ce monde parallèle. Cet espace est une illusion offerte à l'homme comme alternative à la «dead end». Rustin Cohle a un lourd passif avec les drogues en tous genres. Le premier homme qu'il a tué injectait de la méthamphétamine à sa fille pour la «purifier " ${ }^{30}$. Après 1993 et un séjour en hôpital psychiatrique, Rustin se désintoxique. Il arrête toutes substances modificatrices de conscience ; il affirme dans l'épisode pilote (en 1995) «I don't drink ». Cette décision lui permet, selon ses propres mots, de se « réconcilier 
avec sa nature $»^{31}$. La drogue est donc explicitement présentée dans True Detective comme une échappatoire, un refus de l'homme de se confronter avec la Nature et sa nature.

La série propose la religion et la foi comme un autre refuge, permettant à l'homme d'échapper à sa nature. Martin Hart appuie cette thèse dans l'épisode $3:$ il considère la foi religieuse comme un rempart au caractère sauvage de l'homme, et soutient l'hypothèse que, sans éducation religieuse, "it'd be a fucking freak show of murder and debauchery " $^{32}$. En ce sens, il fait sienne la dichotomie classique entre Nature et Culture.

Deux autres illusions apparaissent dans la série, comme les derniers retranchements de l'homme en butte avec sa nature : il s'agit du rêve et de la folie. Piégés par le caractère inéluctable de leur nature, certains personnages refusent simplement ce fardeau. Ils nient leur humanité et créent de nouvelles cosmogonies: c'est le cas de Reggie Ledoux ou d'Errol Childress. La cosmogonie illustrée dans True Detective propose à l'homme de se rapprocher de l'animalité. L'évocation de l'homme-animal est très prégnante dans la série : elle passe par la figure mythique du $"$ roi cerf $»^{33}$, ainsi que par celle des masques d'animaux de la célébration du Mardi gras. Y a-t-il des hommes sous ces masques d'animaux ou bien des animaux sous des masques d'humains? La famille Tuttle est l'emblème de ce jeu de masques. Certains attributs animaux sont reconnus comme des atouts chez un bon enquêteur et la série joue aussi de cette ambiguïté de la part animale de l'homme. En effet, le plan séquence de la fin de l'épisode 4 donne à voir un déchaînement de violence, où la frontière entre nature humaine et bestialité est ténue.

Si la Nature est une impasse, l'unique solution qui s'offre à l'humain est le suicide. Rustin propose cette voie dès l'épisode 1 . Plus tard il conseillera à une mère infanticide de mettre fin à ses jours ${ }^{34}$ et enfin, dans l'épisode 7 , il se présentera lui même comme prêt à « en finir » en bouclant l'enquête, mais aussi le "circle of violence and degradation " $^{35}$ que représente son existence.

Dans True Detective l'humanité apparait comme le jouet d'une entité supérieure. Rustin décrit le travail de l'enquête comme une errance. Pourtant, le caractère particulier de celle-ci révèle plutôt une impasse. Le choix de revenir périodiquement sur des plans d'ensemble en plongée où les personnages, dans une minuscule voiture, sont cernés par l'eau et la végétation, donne à ressentir cet enfermement réel et symbolique. Les hommes apparaissent alors comme des cobayes, jouets de quelque divinité. Cette posture icarienne du regard permet de replacer sans cesse les actions dans un espace circonscrit. Ce regard omniscient juge les personnages. Bien qu'ils aient connaissance de leur destination, ils n'en demeurent pas moins perdus dans une dimension qui les dépasse. Rustin Cohle attribue la subjectivité de ce point de vue à une entité supérieure qu'il nomme «l'éternité ». Ainsi, dans l'épisode 5, il explique : «See, everything outside our dimension, that is eternity, eternity looking down on us. ${ }^{36}$ » 


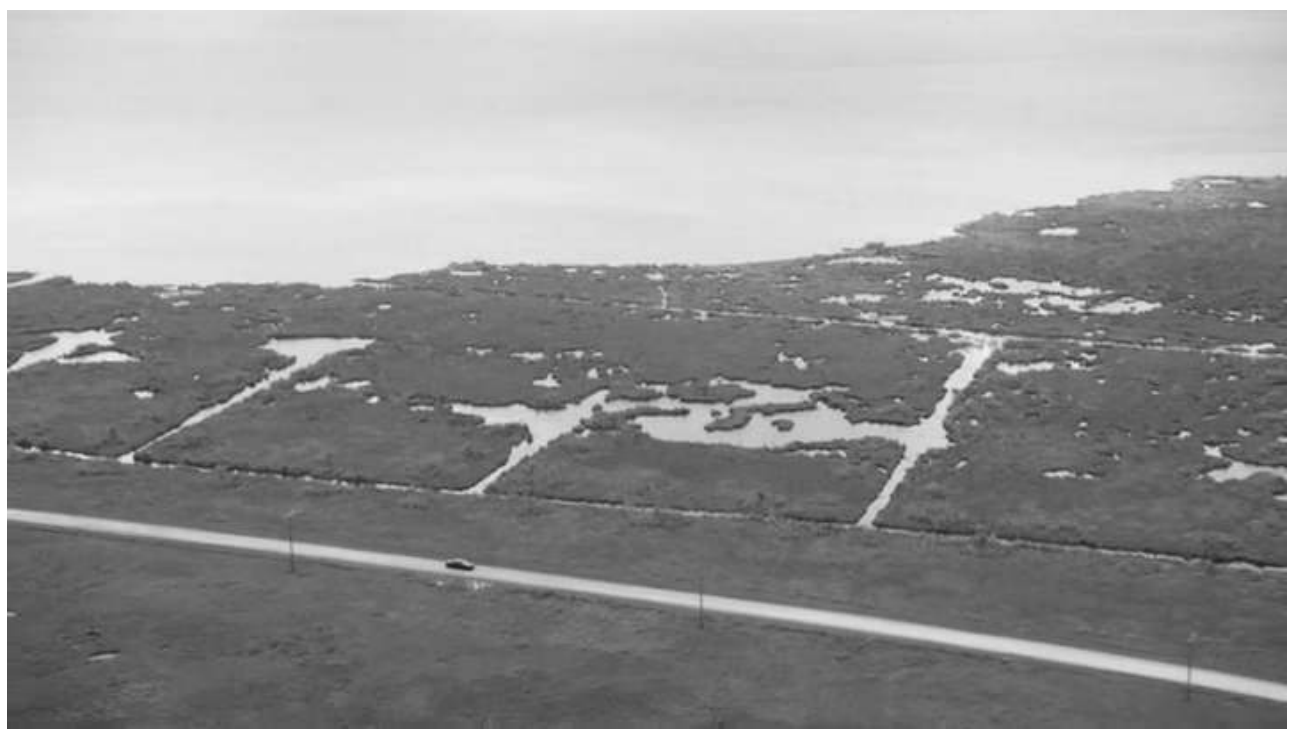

Fig 5 : Posture icarienne du regard 1

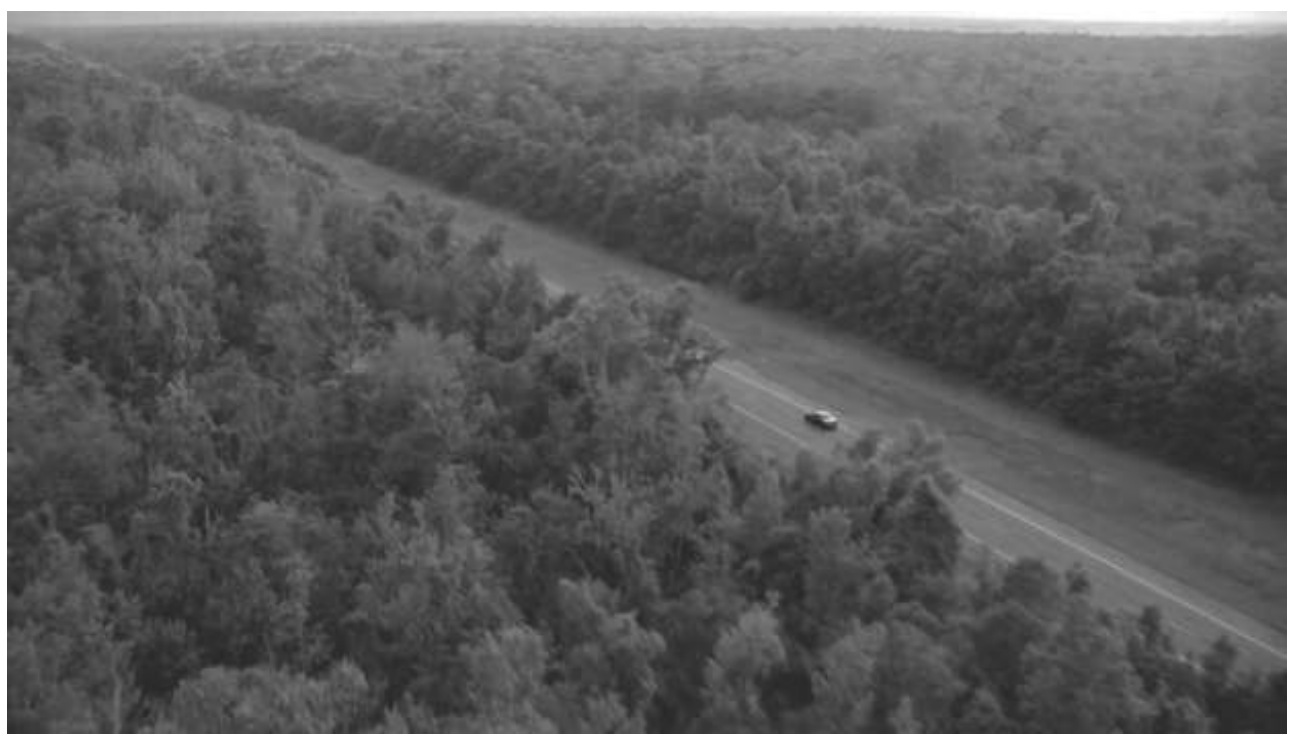

Fig 6 : Posture icarienne du regard 2

28 L'analyse de l'éternité (qui pourrait aussi bien se nommer «Nature $\left.{ }^{37} »\right)$, proposée par Rustin, met en évidence l'importance de ces prises de vues aériennes. Des routes d'une rectitude absolue découpent l'image. Elles renvoient à une perception linéaire du temps et accompagnent les arcs du récit. Pourtant l'éternité, elle, voit un cercle, un espacetemps sans cesse recommencé. Cette nature close, à laquelle on ne peut échapper, embrasse les personnages de True Detective. Dans le dernier épisode de la série, Rustin exprime l'absence de réelle finitude de leur quête. En effet, tous les responsables des disparitions de femmes et d'enfants n'ont pas été arrêtés.

29 La séquence ${ }^{38}$ suivante est consacrée à un retour sur les lieux majeurs de leur parcours, reprenant la posture icarienne. Cette séquence s'achève par un retour aux champs de canne à sucre, où le corps de Dora Lange est découvert dans l'épisode pilote: l'image conclut un cycle.

30 L'espace-temps dans la série renvoie à une forme de purgatoire. Dans l'épisode 5, Rustin Cohle explique aux enquêteurs sa perception d'un temps cyclique et perpétuellement 
identique pour l'humanité et conclut son raisonnement par: "You're trapped by that nightmare you keep waking up into $» .{ }^{39}$ La vision de Rust est celle d'un monde-purgatoire, où nos choix sont illusoires et n'ont aucune réelle finalité. D'une certaine façon, le personnage de Rustin est un naturaliste ${ }^{40}$ déçu par les défaillances de «l'ordre naturel ». Il est à la recherche d'une nature qui soit porteuse d'ordre et de nécessité, une nature gouvernée par une finalité. La conception stoïcienne de l'Éternel retour, défendue notamment par Nietzsche ${ }^{41}$ est un des piliers structurels de la série. La Nature-temps enferme l'homme dans un cycle.

La symbolique du piège est très prégnante dans la série. La séquence finale de l'épisode 5 est, en ce sens, une mise en abîme intéressante. Rustin regarde au travers d'un «bird trap ", alors qu'il est lui même piégé par l'encadrement de la fenêtre. La subjectivité de la caméra semble appartenir à un être omniscient, qu'on le nomme " Nature ", " éternité " ou « divinité ».

Dans un article consacré au paysage comme moment narratif, André Gardies ${ }^{42}$ définit les différentes résonances que peut avoir un plan-paysage dans la dramaturgie cinématographique. Il évoque notamment le «paysage-drame» qui intervient physiquement dans la mise en scène et «participe aux stratégies discursives» du film. Cette appellation semble tout à fait pertinente pour qualifier le travail de Nic Pizzolatto et Cary Fukunaga dans la série True Detective. Les deux premiers longs métrages ${ }^{43}$ du jeune réalisateur de la série présentaient déjà une attention particulière aux espaces naturels et à la composition de plans-paysages.

\section{Conclusion}

Dans la série True Détective, la Nature place les hommes dans une impasse physique et métaphysique. Les singularités géographiques et historiques de la Louisiane remettent en cause toutes les frontières "naturelles »: La vie et la mort, la terre et l'eau, ainsi que le passé et le présent.

La Nature bénéficie dans la diégèse d'une fonction actantielle majeure. En effet, Nic Pizzolato propose une entité qui se présente pareille à l'inscription du temple d'Isis, par ces mots : "Je suis la totalité de ce qui est, qui était et qui sera, et aucun mortel n'a soulevé mon voile $»^{44}$. La Nature dans le récit est une présence qui embrasse la totalité de l'existant, ; elle est origine, principe et cause.

Les réflexions de Ricciotto Canudo ${ }^{45}$ sur la thématique de la nature dans les films, l'amène a considérer son traitement comme un gage de réussite de l'œuvre. Il soutient: «A l'écraniste échoit ce rôle suprême de fondre la vie des hommes dans la vie des milieux ${ }^{46}$. Pour lui, la Nature ne peut être réduite à une enveloppe extérieure, à un simple décor. Le lien symbiotique de celle-ci au drame et aux sentiments des personnages est fondamental. L'espace propose une ambiance, mais peut aller bien au-delà. En effet, il arrive que la Nature se mue en réelle force, une force qui s'exerce sur les personnages. Alors, elle devient un sujet à part entière, le "Personnage-Nature ", un protagoniste polymorphe. La nature est à la fois le sujet et un principe cohérent dans lequel les autres protagonistes existent et acquièrent une réalité.

Dans la série True Detective, la Nature par sa force de vie (et de mort) enserre les personnages. Cette Nature tant physique que métaphysique "piège » l'homme dans l'Histoire. Les détectives Rustin Cohle et Martin Hart regardent en 2012 leur passé et le 
reconstruisent au prisme de leur mémoire. La structure même du récit enferme les protagonistes. La seule issue pour conclure leur errance dans cette Nature-temps forclose est la transcendance divine.

\section{NOTES}

1. http://www.forumdesimages.fr/les-programmes/toutes-les-rencontres/rencontre-avec-nicpizzolatto, consulté le 10 juillet 2016.

2. Angel Heart, Alan Parker, 1987.

3. Louisiana Story, Robert J. Flaherty, 1948.

4. Un tramway nommé désir, Elia Kazan, 1951.

5. Caussarieu, Morgane. Vampire et bayous, sexe, sang et décadence: La résurrection du mythe en Louisiane, Saint Laurent d'Oingt : Éd. Mnémos, 2013.

6. Représentation sociale : «Forme de connaissance, socialement élaborée et partagée, ayant une visée pratique et concourant à la construction d'une réalité commune à un ensemble social » in Jodelet, Denise (dir.). Les représentations sociales. Paris : Presses Universitaires de France, 1989. p. 53.

7. Le climat subtropical humide de la Louisiane donne des températures annuelles entre 20 et $21^{\circ}$

$\mathrm{C}$, le cycle des saisons y est très peu marqué.

8. Denuziere, Maurice. La dix-huitième étoile, Histoire de la Louisiane américaine. Paris : Fayard, 2013. p. 518.

9. Cf. Episode 3. (00:49:00) «Par ici »

10. Cf. Episode 1. (00:53:22)

11. Cf. Épisode 2. (00:05:39)

12. Cf. Episode 1. (00:14:13) «Les gens par ici, c'est comme s'ils ignoraient que le monde extérieur existe "

13. White trash: Terme dépréciatif qui désigne la classe populaire blanche du sud rural des EtatsUnis. Ces populations vivent dans une très grande pauvreté, en marge de la société mondialisée. Ces groupes sont désignés comme dangereux et réfractaires à toute autorité qu'elle soit politique, juridique ou morale.

14. Cf. Episode 2. (00:04:44) "Why wouldn't a father bathe his own child?», « Pourquoi un père ne baignerait-il pas son propre enfant? » et Episode 2. (00:32:23)

15. Cf. Episode 8. (00:05:00)

16. Cf. Episode 3. (00:40:02)

17. Cf. Episode 2. (00:55:40)

18. http://www.forumdesimages.fr/les-rencontres/toutes-les-rencontres/rencontre-avec-nicpizzolatto, consulté le 10 mars 2016

19. L'impact des plus en plus important de l'activité humaine sur la biosphère a donné lieu au concept d'anthropocène.

20. Cf. Episode 1. (00:14:25) « Il y a toutes sortes de ghettos dans le monde », « le monde entier est un ghetto »

21. Contraire à la Nature dans ses diverses acceptions normatives.

22. La nature-essence : propriétés de l'être dont il est question.

23. Cf. Episode 1. (00:34:39) 
24. La nature humaine se définit en opposition à la culture. Elle englobe l'ensemble des caractères qui définissent l'homme, considérés comme innés, comme indépendants à la fois des déterminations biologiques et des déterminations sociales, historiques, culturelles. (Cf. http:// www.cnrtl.fr/lexicographie/nature )

25. Cf. Episode 1. (00:16:18) \& Episode 3. (00:35:12)

26. Cf. Episode 1. (00:16:53)

27. Adepte du mouvement de réveil protestant, prosélyte qui ont comme objectif fort la réanimation de la foi assoupie.

28. Cf. Episode 6. (00:15:30) « Toute ma vie j'ai voulu être plus proche de Dieu, mais la seule chose dont j'ai été proche, c'est le silence ».

29. Cf. Episode 6. (00:39:57) «C'est difficile de se fier à un homme incapable de résister à une bière ».

30. Cf. Episode 2. (00:37:10)

31. Cf. Episode 1. (00:30:25)

32. Cf. Episode 3. (00:05:54) "Ce serait un festival de meurtres et de débauche. "

33. Le roi cerf ou dieu cerf Cernunnos est une figure du panthéon celtique et gaulois. Charniguet, Alexis et Lombard-Jourdan, Anne. Cernunnos, dieu Cerf des Gaulois. Paris : Éd. Larousse, 2009.

34. Cf. Episode 6. (00:21:40)

35. Cf. Episode 7. (00:47:33) «Cercle de violence et de décadence.»

36. Cf. Episode 5. (00:33:24) «Vous voyez, tout ce qui est extérieur à notre dimension, c'est l'éternité, l'éternité qui nous regarde d'en haut.»

37. Le terme "nature » est polysémique, ce qui le rend d'un abord complexe. Les géographes la définissent comme l'ensemble des écosystèmes que l'homme a peu altéré et comme le refuge de la biodiversité. Le mot est ici pris dans son acception philosophique se rattachant à une vision panthéiste du monde. La nature est un principe caché, immatériel de production et de génération ayant sa finalité propre.

38. Cf. Episode 8. (00:46:28) à (00:47:34)

39. Cf. Episode 5. (00:41:35) « Tu est piégé par ce cauchemar dans lequel tu ne cesses de te réveiller. "

40. Attaché au concept de Nature. Cf. Rosset, Clément. L'anti-nature. Paris : Quadrige (5eme Edition), 2011.

41. Concept présent dans Le Gai savoir puis dans Ainsi parlait Zarathoustra.

42. Mottet, Jean (dir.). Les paysages du cinéma. Seyssel : Champ Vallon, 1999. P. 145-150

43. Sin Nombre (2009), Jane Eyre (2011)

44. Château, Jean-Yves, " Nature », in De Baecque, Antoine et Chevalier Philippe. Dictionnaire de la pensée du cinéma. Paris : PUF, 2012. p. 491- 495.

45. Canudo, Ricciotto. L'usine aux images. Genève : Office central d'édition, 1927.

46. Ibid.

\section{AUTEUR}

\section{MAYLIS ASTÉ}

Maylis Asté obtient un master de recherche en histoire puis poursuit son cursus universitaire en intégrant l'ESAV (École Supérieure d'Audiovisuel de Toulouse), où elle obtient un Master 
Professionnel en Réalisation. A la croisée de ces parcours, elle débute en 2013 un doctorat en histoire consacré aux représentations de la ruralité dans le cinéma français, des années 1990 à 2010. Elle est membre de l'équipe « Dynamiques Rurales» du LISST à l'Université Toulouse 2. 